5 Research Square
Preprints are preliminary reports that have not undergone peer review.

They should not be considered conclusive, used to inform clinical practice, or referenced by the media as validated information.

\title{
Evaluation of the Consequences of Double Ovarian Stimulation Through Shanghai Protocol in Patients With Poor Ovarian Respond
}

Mahvash Zargar ( $\nabla$ dr.mahvashzargar199@yahoo.com )

Ahvaz Jondishapour University of Medical Sciences

\section{Zahra Pourjafar}

Ahvaz Jundishapur University of Medical Sciences

Shabnam Salemi

Ahvaz Jundishapur University of Medical Sciences

Mojgan Barati

Ahvaz Jundishapur University of Medical Sciences

\section{Research Article}

Keywords: Ovarian Stimulation, Luteal phase, Follicular phase, Letrozole, Clomid

Posted Date: February 16th, 2022

DOI: https://doi.org/10.21203/rs.3.rs-1283105/v1

License: (c) (i) This work is licensed under a Creative Commons Attribution 4.0 International License.

Read Full License 


\section{Abstract}

Purpose: This prospective study is aimed at assessing the consequences of double ovarian stimulation through Shanghai protocol in women diagnosed as poor ovarian responders (POR), compared with the conventional IVF.

Methods: A total of 84 women diagnosed as POR were divided into two groups, including those who underwent conventional IVF protocol $(\mathrm{N}=42)$ and or Shanghai protocol $(\mathrm{N}=42)$. In Shanghai protocol, ovarian stimulation was firstly done in the early follicular phase and secondly in the luteal phase of the same ovarian cycle after the first oocyte retrieval. For primary ovarian stimulation, Letrozole $2.5 \mathrm{mg} /$ day and Clomid $25 \mathrm{mg}$ /day were administered for 4 consecutive days from cycle day 2 onwards.

Results: After treatment, 1 to 5 oocytes were retrieved in 34 women (85\%) underwent Shanghai protocol, which was significantly higher than its rate in the conventional IVF group [26(61.90\%), $p=0.02$ ]. Subsequently, 33 women (82.50\%) could develop 61 live embryos at the end of the second stimulation, which was significantly higher than its rate in another group [ $50 \%, P=0.002]$. The rates of Metaphase II oocytes $(60.82 \%)$ and high-quality embryos $(93.44 \%)$ obtained during Shanghai protocol were significantly higher than their rates in conventional IVF $(45.24 \%$ and $68.42 \%$, respectively, $p<0.05)$. Eventually, the clinical pregnancy success rate was significantly higher in women underwent Shanghai protocol $(42.50 \%)$ than those underwent conventional IVF $(19.05 \%)[p=0.03]$.

Conclusion: POR women underwent the double ovarian stimulation during Shanghai protocol-based IVF achieved the higher rates of high-quality retrieved oocytes, embryos, and successful pregnancy, compared with those who had underwent the conventional IVF.

\section{Introduction}

Infertility has always been one of the main human problems inducing many psychological and social consequences for couples and families. Infertility rates have been rising in recent decades and affecting about 10_15\% of couples worldwide [1, 2]. IVF is the most reasonable assisted reproductive technology (ART) treatment option for women suffering from infertility due to aging and or other causes. IVF success over the past years has increased that this depends on the IVF treatment method and patients' characteristics [3].

The quality of ovarian response during stimulation for IVF cycle is a matter of debate [4]. Controlled ovarian stimulation (COS) is a form of prerequisite medication therapy for ART, which can provide a sufficient number of transferable embryos and minimize the risk of ovarian overstimulation $[5,6]$. There is an independent correlation between the optimal number of oocytes and ovarian response following COS with live birth rates following IVF. Nevertheless, the live birth rate has not been affected by the number of oocytes in women undergoing COS for their first IVF cycle; however, the higher the oocyte yield, the possibility of live birth is more after using all frozen embryos [7]. 
A poor ovarian response (POR) was seen in about $5-24 \%$ of patients undergoing ovarian stimulation for IVF [4]. In the last decade, many studies have been conducted on POR management methods, yet the treatment of these patients remains a challenging issue for physicians due to insufficient evidence to determine the best protocol $[8,9]$. A new protocol named the Shanghai protocol was proposed by Kuang et al. (2014) for the treatment of patients with poor ovarian response, which is based on double stimulations during the follicular and luteal phases using letrozole, clomid, gonadotropin-releasing hormone (GnRH), and human menopausal gonadotropin (hMG) agonists [4].

To date, few studies have been done on the effectiveness of Shanghai protocol $[4,9,10]$. Accordingly, this study was performed to evaluate the outcomes of the double ovarian stimulation through Shanghai protocol in women diagnosed as poor ovarian responders referred to Imam Khomeini Hospital in Ahvaz, compared with the conventional IVF protocol.

\section{Methods}

\subsection{Study design and patients}

In this prospective case-control study, a total of 84 women with a POR divided into two groups, including those who underwent conventional IVF protocol $(\mathrm{N}=42)$ and or IVF treatment based on the Shanghai protocol $(\mathrm{N}=42)$. All procedures in this study involving human participants were in accordance with the ethical standards of the national research committee and with the 2008 Helsinki declaration and its later amendments or comparable ethical standards. This study was approved by the ethics committee of Ahvaz Jundishapur University of Medical Sciences with Ethical Code:

IR.AJUMS.HGOLESTAN.REC.1400.019.

Patients who have at least two of the following criteria were considered as poor ovarian responders: AMH $<1 \mathrm{ng} / \mathrm{mL}$, a basal serum follicle stimulating hormone (FSH) concentration between 10 and $19 \mathrm{IU} / \mathrm{l}$, a history of POR and treatment with one protocol that resulted in less than 3 oocytes, and antral follicle count $<5$. Women with ovarian failure or basal FSH $>20 \mathrm{IU} / \mathrm{I}$ or no antral follicle on ultrasound, endometriosis grade 3 or higher, and any contraindications to ovarian stimulation treatment were excluded from the study. All the patients signed the informed consent and voluntary participated in this study.

\subsection{Treatment protocols}

As shown in Fig. 1, 42 patients were treated by conventional IVF protocol [1] while others were undergoing double ovarian stimulation with Shanghai protocol [4]. In this regard, to obtain more oocytes and embryos, double ovarian stimulation was firstly done in the early follicular phase and secondly in the luteal phase of the same ovarian cycle after the first oocyte retrieval. According to the Shanghai protocol, in order to primary ovarian stimulation, Letrozole $2.5 \mathrm{mg}$ /day and Clomid $25 \mathrm{mg} /$ day were administered for 4 consecutive days from cycle day 2 onwards. On the sixth day, Letrozole was replaced with human menopausal gonadotropin (HMG) while Clomid continued. HMG injection (150 IU) for patients was 
started every other day from cycle day 6 onwards. Follicular monitoring was performed using ultrasonography and measurement of serum estrogen concentration to determine the administration time of gonadotropin releasing hormone $(\mathrm{GnRH})$ agonist. Follicular monitoring was performed using ultrasonography and measurement of serum estrogen concentration to determine the administration time of $\mathrm{GnRH}$ agonist. When one or two pioneer follicles reached $18 \mathrm{~mm}$ in diameter, the final stage of oocyte maturation was stimulated using $0.1 \mathrm{mg} / \mathrm{mL}$ Triptorelin. Ultrasound-guided oocyte retrieval was done 3236 hours after $\mathrm{GnRH}$ agonist administration. Two to three days after collecting the first stage oocytes, the second stimulation was started with Letrozole $2.5 \mathrm{mg} /$ day and HMG $225 \mathrm{IU} /$ day. All high quality embryos were cryopreserved by standard methods, and eventually, the frozen embryo transfer (FET) protocol was implemented for all patients.

\subsection{Evaluation of treatment outcomes}

Patients' demographic characteristics, e.g., age, marriage duration, gravity, history of abortion and/or live birth, type of infertility, duration of infertility as well as the number of IVF and IVF failures were collected by interviewing with patient and/or from patients' medical records.

The quality of embryos was determined based on the number of blastomeres and embryo cleavage stage (or the amount of embryo fragmentation) as grades 1 to 4 [11]. Eventually, the cancellation rate of the treatment cycle and the rate of clinical pregnancy were assessed according to the rate of embryo transfer.

\subsection{Statistical analysis}

SPSS software version 26 (SPSS Inc., Chicago, III., USA) was used for statistical analysis. The normality of variables was checked by the Kolmogorov-Smirnov test. Categorical and continuous variables presented as frequency or percentages and the mean \pm SD (standard deviation), respectively. The chisquare test was used to compare the categorical variables while the continuous variables were compared through an independent t-test and/or Mann-Whitney $U$ test. $P$ value less than 0.05 was considered to be statistically significant.

\section{Results}

As shown in Fig. 2, from a total of 42 patients under shanghai protocol treatment, 2 cases were excluded from the study due to cancellation of participation and/or evaluation failure of the number of produced follicles. Four patients in both groups did not produce any antral follicles, and respectively, 36 and 38 women in shanghai group and conventional IVF group produced one to eight follicles.

Table 1 summarized demographic and basic medical characteristics of women indicating that there was no significant difference between two treated groups in terms of age, gravidity, parity, history of abortion, cause of infertility, duration of infertility, and IVF history $(p>0.05)$. 
Table 1

Demographic and basic medical characteristics of women underwent Shanghai protocol and/or the conventional IVF protocol.

\begin{tabular}{|c|c|c|c|}
\hline \multirow[t]{3}{*}{ Variables } & \multicolumn{2}{|c|}{ Mean \pm S.D (Min-Max) } & \multirow[t]{3}{*}{$P$ value } \\
\hline & women underwent & women underwent & \\
\hline & Shanghai protocol & conventional IVF protocol & \\
\hline Age (year) & $35.31 \pm 4.29$ & $35.76 \pm 6.30$ & 0.60 \\
\hline \multicolumn{3}{|l|}{ Gravidity } & \multirow[t]{4}{*}{0.64} \\
\hline 0 & $26(61.90 \%)$ & $23(54.76 \%)$ & \\
\hline 1_2 & $14(33.33 \%)$ & $15(35.71 \%)$ & \\
\hline 3_4 & $2(4.76 \%)$ & $4(9.53 \%)$ & \\
\hline \multicolumn{4}{|l|}{ Parity } \\
\hline 0 & $28(66.67 \%)$ & 25 (59.53\%) & \\
\hline 1 & $7(16.67 \%)$ & $9(21.43 \%)$ & 0.78 \\
\hline 2 & $7(16.67 \%)$ & $8(19.04 \%)$ & \\
\hline History of abortion (Min-Max: 1_2) & $1.50 \pm 0.51$ & $1.57 \pm 0.54$ & 0.58 \\
\hline \multicolumn{4}{|l|}{ Cause of infertility: } \\
\hline Male infertility & $26(61.90 \%)$ & $20(47.62 \%)$ & \\
\hline Female infertility & $11(26.20 \%)$ & $13(30.95 \%)$ & 0.55 \\
\hline Male-Female infertility & $4(9.50 \%)$ & $7(16.66 \%)$ & \\
\hline Unexplained & $1(2.40 \%)$ & $2(4.76 \%)$ & \\
\hline Duration of infertility (1_15 Year) & $4.95 \pm 2.86$ & $5 \pm 0.70$ & 0.57 \\
\hline \multicolumn{4}{|l|}{ Number of IVF: } \\
\hline Not IVF history & $23(54.76 \%)$ & $19(45.24 \%)$ & \\
\hline 1 & $11(26.20 \%)$ & $12(28.57 \%)$ & 0.81 \\
\hline 2 & $4(9.50 \%)$ & $5(11.91 \%)$ & \\
\hline 3 & $4(9.50 \%)$ & 6 (14.28\%) & \\
\hline
\end{tabular}

After double ovarian stimulation, 1 to 5 oocytes $(2.43 \pm 1.33)$ were retrieved in 34 women (85\%), which was significantly higher than its rate in the conventional IVF treated group [26 cases $(61.90 \%), p=0.02$ ]. Subsequently, from a total of 42 women under conventional IVF treatment, only 21 women succeeded in 
producing 1-3 live embryos, and so, their success rate of embryo production was $50 \%$. But, out of 40 women undergoing shanghai protocol, 33 women had 1 to 3 live embryos and only one case had no embryo; in other words, the success rate of embryo production was $82.5 \%$ which was significantly higher than that of the conventional IVF group $[P=0.002$, Table 2]. 
Table 2

Comparison of consequences of the frozen embryo transfer (FET) cycle through the Shanghai protocol with the conventional protocol.

\begin{tabular}{|c|c|c|c|}
\hline Variables & $\begin{array}{l}\text { Patients under } \\
\text { Shanghai protocol } \\
(n=40)\end{array}$ & $\begin{array}{l}\text { Controls under } \\
\text { conventional IVF } \\
(n=42)\end{array}$ & $\begin{array}{l}P \\
\text { value }\end{array}$ \\
\hline $\begin{array}{l}\text { Number of produced follicles } \\
(0-8)\end{array}$ & $3.10 \pm 1.75$ & $3.15 \pm 1.38$ & 0.66 \\
\hline $\begin{array}{l}\text { Number of Oocytes } \\
(0-5)\end{array}$ & $2.43 \pm 1.33$ & $1.76 \pm 0.93$ & 0.13 \\
\hline $\begin{array}{l}\text { Number of patients with retrieved oocytes } \\
\text { Yes }\end{array}$ & $3485 \%)$ & $26(61.90 \%)$ & 0.02 \\
\hline No & $6(15 \%)$ & $12(38.10 \%)$ & \\
\hline $\begin{array}{l}\text { Quality of retrieved oocytes } \\
\text { [97 oocytes under Shanghai protocol vs } 84 \\
\text { oocytes under conventional IVF]: } \\
\text { Metaphase I }\end{array}$ & $27(27.84 \%)$ & $39(46.43 \%)$ & 0.02 \\
\hline Metaphase II & $59(60.82 \%)$ & $38(45.24 \%)$ & \\
\hline Metaphase III & $11(11.34 \%)$ & $7(8.33 \%)$ & \\
\hline $\begin{array}{l}\text { Number of embryos: } \\
0\end{array}$ & $1(2.94 \%)$ & $5(19.23 \%)$ & 0.07 \\
\hline 1 & $9(26.47 \%)$ & $6(23.07 \%)$ & \\
\hline 2 & $20(58.83 \%)$ & $13(50 \%)$ & \\
\hline 3 & $4(11.76 \%)$ & $2(7.70 \%)$ & \\
\hline Total & 61 & 38 & \\
\hline Embryo Quality Grading: & & & 0.009 \\
\hline Grade 1 & $6(9.84 \%)$ & $3(7.90 \%)$ & \\
\hline Grade 2 & 35 (57.37\%) & $16(42.10 \%)$ & \\
\hline Grade 3 & $16(26.23 \%)$ & 7 (18.42\%) & \\
\hline Grade 4 & $4(6.56)$ & $12(31.58 \%)$ & \\
\hline Total & 61 & 38 & \\
\hline
\end{tabular}




\begin{tabular}{|llll|}
\hline Variables & $\begin{array}{l}\text { Patients under } \\
\text { Shanghai protocol } \\
(\mathbf{n = 4 0 )}\end{array}$ & $\begin{array}{l}\text { Controls under } \\
\text { conventional IVF } \\
(\mathbf{n = 4 2 )}\end{array}$ & $\begin{array}{l}\text { P } \\
\text { value }\end{array}$ \\
\hline Number of patients with live embryos & $33(82.50 \%)$ & $21(50 \%)$ & 0.002 \\
Number of patients without any embryos & $7(17.50 \%)$ & $21(50 \%)$ & 0.40 \\
\hline $\begin{array}{l}\text { Clinical Pregnancy rate in patients who } \\
\text { completed FET cycle (\%) }\end{array}$ & $17(51.51 \%)$ & $8(38.10 \%)$ & \\
$\begin{array}{l}\text { None clinical pregnancy } \\
\text { Success rate of clinical pregnancy in all } \\
\text { evaluated patients }(\%)\end{array}$ & $16(48.49 \%)$ & $13(61.90 \%)$ & \\
$\begin{array}{l}\text { Failed rate of clinical pregnancy in all } \\
\text { evaluated patients }(\%)\end{array}$ & $17(42.50 \%)$ & $8(19.05 \%)$ & \\
\hline
\end{tabular}

Assessment of in-vitro embryo quality showed that the percentage of good to highest quality embryos with grade 1 to 3 in women underwent shanghai protocol (93.44\%) was significantly higher than their percentage in women underwent conventional IVF protocol $(68.42 \% ; p=0.009)$.

Eventually, from a total of 33 women who completed the frozen embryo transfer cycle, 17 cases achieved a clinical pregnancy while only 8 cases of women under conventional IVF treatment got pregnant; the success rate of clinical pregnancy was clinically higher in women underwent Shanghai protocol $(42.50 \%)$ than those underwent conventional IVF (19.05\%) [p=0.03; Table 2].

\section{Discussion}

We found that the numbers and quality of retrieved oocytes, and embryo quality during double ovarian stimulation are considerably higher than those during the conventional IVF protocol. The number of created embryos in patients underwent shanghai protocol were significantly more than those in the conventional IVF protocol. Evaluation of the quality of retrieved oocytes showed that most of the retrieved oocytes were at metaphase II (MII) in both studied groups, and $60.82 \%$ of retrieved oocytes obtained from shanghai protocol were MII oocytes.

Mild stimulation at the follicular phase and subsequent ovarian stimulation at the luteal phase to maximize oocytes retrieval are firstly proposed by Kuang et al [4]. Their pilot study on 38 women with POR showed that the mean number of follicles, retrieved oocytes, and MIl oocytes obtained during shanghai protocol were respectively $3.9,2.6$, and 2.05 , which was close to their values in our study. Their results indicated that the stimulation in the luteal phase led to retrieval of more oocytes in a short period of time. In this regard, our results were confirming their report in which the rate of retrieved oocytes obtained during shanghai protocol were significantly higher than its rate in conventional IVF protocol. Also, the results of Madani et al.'s study showed that the mean number of MII oocytes in the shanghai 
protocol was remarkably higher than previous antagonist protocols [9]. In the study of Zhang [6], the double ovarian stimulation in women with POR resulted in the production of the highest number of oocytes in the shortest time. Our findings on the shanghai protocol were also consistent with the results from Moffat et al.'s pilot study; They reported that the rate of oocytes and blastocysts obtained from double ovarian stimulation was twice their rate in ART by a single ovarian stimulation based on a standard antagonist protocol. This method is also significantly tolerated by women [12]. Also, the results of Liu et al.'s study on women over 38 years with POR showed that in the protocol of double ovarian stimulation in the follicular and luteal phases, the number of retrieved oocytes and live embryos were twice as high as their number in the follicular phase of ovarian stimulation [13]. These results indicate that double ovarian stimulation can increase the chances of getting pregnant by accumulating more oocytes and embryos in a short period of time and can be a useful strategy for older women.

In the present study, from a total of 40 women undergoing shanghai protocol, 33 women could develop 61 live embryos (1 to 3 embryos per person) and only one case had no embryo; in other words, the success rate of embryo production in shanghai protocol (82.5\%) was considerably more than its rate in women under conventional IVF (50\%). Also, the percentage of good to highest quality embryos in women underwent shanghai protocol (93.44\%) was significantly higher than their percentage in women underwent conventional IVF protocol $(68.42 \% ; p=0.009)$. In the study of Madani et al. [9], out of 121 patients underwent double ovarian stimulation, 164 live embryos were obtained from 81 patients (67\%) (1 to 7 embryos per patient) and other patients failed to develop any embryos due to lack of oocytes retrieval in 17 patients and lack of embryo development in 23 patients. In Kuang et al.'s study [4], out of 38 women treated with shanghai protocol based double stimulation, 12 cases did not develop any embryos due to none oocyte retrieval, immature oocytes, unfertilized oocytes, or embryo fragmentation; twenty-six women (68.4\%) could develop 72 live embryos (1 to 6 embryos per person) at the end of the second ovarian stimulation.

Numerous factors are associated with poor ovarian response, and diminished ovarian reserve is the most common cause in both old and young patients. During depletion of ovarian reserve, stimulation of multiple follicular growth remains a problem [14]. Previous evidence showed that folliculogenesis occurs during the menstrual cycle in a wave-like model; in other words, there are multiple follicular recovery waves in a menstrual cycle [15]. In this regard, the shanghai protocol with two-stage stimulation in one cycle provides a new opportunity for the management and treatment of women with diminished ovarian reserves [4]. In the shanghai protocol, the first stimulation normally begins in the early follicular phase while the second stimulation occurs on the first day following the first oocyte retrieval if there are two or more antral follicles [4].

Simultaneously use of clomid and letrozole in the early follicular phase have been suggested by Kuang et al. [4] to increase the ovarian response, which can have a coordinating function. Clomid increases pituitary related secretion of FSH by reducing negative estrogen feedback. On the other hand, letrozole is a potent non-steroidal aromatase inhibitor that inhibits the aromatization of androgens to estrogen and its release from the hypothalamic-pituitary axis caused by estrogen-negative feedback. Such an increase 
in intra-ovarian androgens can stimulates primary follicular growth, and so, improves IVF results [16]. Letrozole has previously been reported as an effective adjuvant agent for the $\mathrm{GnRH}$ antagonist in patients with POR $[17,18]$. In this regard, Bastu et al. [17] examined the consequences of IVF after ovarian stimulation with different doses of gonadotropin with or without letrozole in a clinical trial; they showed that the use of mild stimulation with letrozole is as effective as stimulation with higher doses of lone gonadotropin. Recombinant gonadotropins (FSH and LH) have been used instead of letrozole or clomid in other recent new ovarian double-stimulation strategies evaluated by Ubaldi et al. [19] And Cardoso et al. [20]; five days after oocyte retrieval, luteal phase stimulation began, which eventually led to the retrieval of more euploid oocytes and blastocytes in a menstrual cycle.

In the present study, among 33 women who completed FET cycle, 17 cases achieved a clinical pregnancy, which was higher than the clinical pregnancy rate in those underwent the conventional IVF treatment. In Cardoso et al.'s study, the clinical pregnancy rate was $22 \%$ in women over the age of 35 with POR treated with the double ovarian stimulation and FET, which is less than the success rate of pregnancy in our study (42.50\%). Unlike the shanghai protocol, they used FSH and LH in the first stimulation and HMG in the second stimulation [21], implies that Clomid and Letrozole may probably more effective than recombinant gonadotropins.

Our prospective comparative study is one of the few studies evaluating the consequences of Shanghai protocol in patients with poor ovarian response, and showed desirable results in attaining higher rates of retrieved oocytes, high quality embryos, and clinical pregnancy. Nevertheless, this comparative study was merely observational; so, unlike the randomized clinical trials, it may not able to minimize the effect of confounding factors, avoid bias in allocation to groups, and detect small to moderate effects that may be clinically important. For example, we have not assessed the miscarriage.

\section{Conclusion}

Findings of our prospective study suggested that women with poor ovarian response underwent the double ovarian stimulation in the follicular and luteal phase of the ovarian cycle (Shanghai strategy) achieved the higher rates of high-quality retrieved oocytes (60.82\%), moderate to high quality embryos $(82.50 \%)$, and successful clinical pregnancy $(42.50 \%)$, compared with women who had underwent conventional IVF protocol. Nevertheless, randomized clinical trials are needed to more accurately evaluate the clinical utility and economical aspect of this method in comparison to other ART treatments.

\section{Declarations}

\section{Acknowledgments}

The present article is taken from Dr. Zahra Pourjafar's residency dissertation with the research project number FIRC-0005 in the medical faculty of Ahvaz Jundishapur University of Medical Sciences with Ethical Code: IR.AJUMS.HGOLESTAN.REC.1400.019. 


\section{Conflicts of interest}

There are no conflicts of interest for this study.

\section{Availability of data and material}

Data supporting the findings of this study are available from the corresponding author on request.

\section{Ethics approval}

All procedures performed in studies involving human participants were in accordance with the ethical standards of the national research committee and with the 2008 Helsinki declaration and its later amendments or comparable ethical standards. This study was approved by the ethics committee of Ahvaz Jundishapur University of Medical Sciences with Ethical Ethical Code: IR.AJUMS.HGOLESTAN.REC.1400.019.

\section{Consent to participate}

The decision to participate in this study was completely voluntary for all patients. At the end of the study, all participants had free access to their laboratory and clinical results.

\section{Consent for publication}

All individuals involved in this clinical trial are aware of the planned publication and had given their consent at study initiation.

\section{Author Contribution}

Z. M: Conceptualization, methodology, supervision and funding acquisition, investigation, writing original draft, review and editing; P. Z: investigation, Data curation, writing \& review \& editing; S.S: investigation, B.M: Clinical Consultation

\section{References}

1. Zargar M, Pazhouhanfar R, Najafian M, Moradi Choghakabodi P (2021) Effects of intrauterine autologous platelet-rich plasma infusions on outcomes in women with repetitive in vitro fertilization failures: a prospective randomized study. Clin Exp Obstet Gynecol 48(1):179-184.

2. Rooney KL, Domar AD (2018) The relationship between stress and infertility. Dialogues Clin Neurosci 20(1):41-47.

3. Zargar M, Dehdashti S, Najafian M, Choghakabodi PM (2021) Pregnancy outcomes following in vitro fertilization using fresh or frozen embryo transfer. JBRA Assist Reprod 25(4):570-574.

4. Kuang Y, Chen Q, Hong Q, Lyu Q, Ai A, Fu Y, et al (2014) Double stimulations during the follicular and luteal phases of poor responders in IVF/ICSI programmes (Shanghai protocol). Reprod Biomed 
Online 29(6):684-91.

5. Vuong LN, Dang VQ, Ho TM, Huynh BG, Ha DT, Pham TD, et al (2018) IVF Transfer of Fresh or Frozen Embryos in Women without Polycystic Ovaries. N Engl J Med 378(2):137-147.

6. Zhang J (2015) Luteal phase ovarian stimulation following oocyte retrieval is it helpful for poor responders. Reprod Biol Endocrinol 13:76.

7. Drakopoulos P, Blockeel C, Stoop D, Camus M, de Vos M, Tournaye H, et al (2016) Conventional ovarian stimulation and single embryo transfer for IVF/ICSI. How many oocytes do we need to maximize cumulative live birth rates after utilization of all fresh and frozen embryos? Hum Reprod 31(2):370-76.

8. Drakopoulos P, Bardhi E, Boudry L, Vaiarelli A, Makrigiannakis A, Esteves SC, et al (2020) Update on the management of poor ovarian response in IVF: the shift from Bologna criteria to the Poseidon concept. Ther Adv Reprod Health 14:2633494120941480.

9. Madani T, Hemat M, Arabipoor A, Khodabakhshi SH, Zolfaghari Z (2019) Double mild stimulation and egg collection in the same cycle for management of poor ovarian responders. J Gynecol Obstet Hum Reprod 48(5):329-333.

10. Luo Y., Sun L., Dong M., Zhang X., Huang L., Zhu X, et al (2020) The best execution of the DuoStim strategy (double stimulation in the follicular and luteal phase of the same ovarian cycle) in patients who are poor ovarian responders. Reproductive Biology and Endocrinology 18:102.

11. Madani T, Hemat M, Arabipoor A, Khodabakhshi SH, Zolfaghari Z (2019) Double mild stimulation and egg collection in the same cycle for management of poor ovarian responders. J Gynecol Obstet Hum Reprod 48(5):329-333.

12. Moffat R, Pirtea P, Gayet V, Wolf JP, Chapron C, de Ziegler D (2014) Dual ovarian stimulation is a new viable option for enhancing the oocyte yield when the time for assisted reproductive technnology is limited. Reprod Biomed Online 29(6):659-61.

13. Liu C, Jiang H, Zhang W, Yin H (2017) Double ovarian stimulation during the follicular and luteal phase in women $\geq 38$ years: a retrospective case-control study. Reprod Biomed Online 35(6):678-684.

14. Rasool S, Shah D (2017) Fertility with early reduction of ovarian reserve: the last straw that breaks the Camel's back. Fertil Res Pract 3:15.

15. Baerwald AR, Adams GP, Pierson RA (2012) Ovarian antral folliculogenesis during the human menstrual cycle: a review. Hum Reprod Update 18:73-91.

16. Garcia-Velasco JA, Moreno L, Pacheco A, Guillén A, Duque L, Requena A, et al (2005) The aromatase inhibitor letrozole increases the concentration of intraovarian androgens and improves in vitro fertilization outcome in low responder patients: a pilot study. Fertil Steril 84(1):82-7.

17. Bastu E, Buyru F, Ozsurmeli M, Demiral I, Dogan M, Yeh J (2016) A randomized, singleblind, prospective trial comparing three different gonadotropin doses with or without addition of letrozole during ovulation stimulation in patients with poor ovarian response. Eur J Obstet Gynecol Reprod Biol 203:30-4. 
18. Yarali H, Esinler I, Polat M, Bozdag G, Tiras B (2009) Antagonist/letrozole protocol in poor ovarian responders for intracytoplasmic sperm injection: a comparative with the microdose flare-up protocol. Fertil Steril 92(1):231-5.

19. Ubaldi FM, Capalbo A, Vaiarelli A, Cimadomo D, Colamaria S, Alviggi C, et al (2016) Follicular versus luteal phase ovarian stimulation during the same menstrual cycle (DuoStim) in a reduced ovarian reserve population results in a similar euploid blastocyst formation rate: new insight in ovarian reserve exploitation. Fertil Steril 105(6):1488-1495.e1.

20. Cardoso MCA, Evangelista A, Sartório C, Vaz G, Werneck CLV, Guimarães FM, et al (2017) Can ovarian double-stimulation in the same menstrual cycle improve IVF outcomes? JBRA Assist Reprod 21(3):217-221.

\section{Figures}

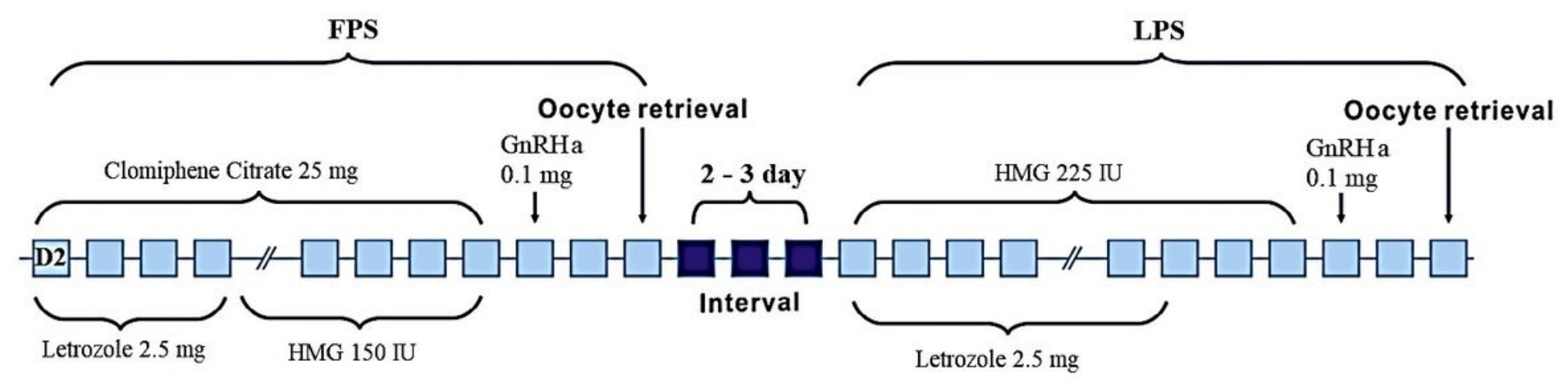

\section{Figure 1}

Steps of double ovarian stimulation with Shanghai protocol. FPS: follicular-phase ovarian stimulation; LPS: luteal-phase ovarian stimulation; GnRHa: gonadotrophin-releasing hormone agonist; HMG, human menopausal gonadotrophin. 


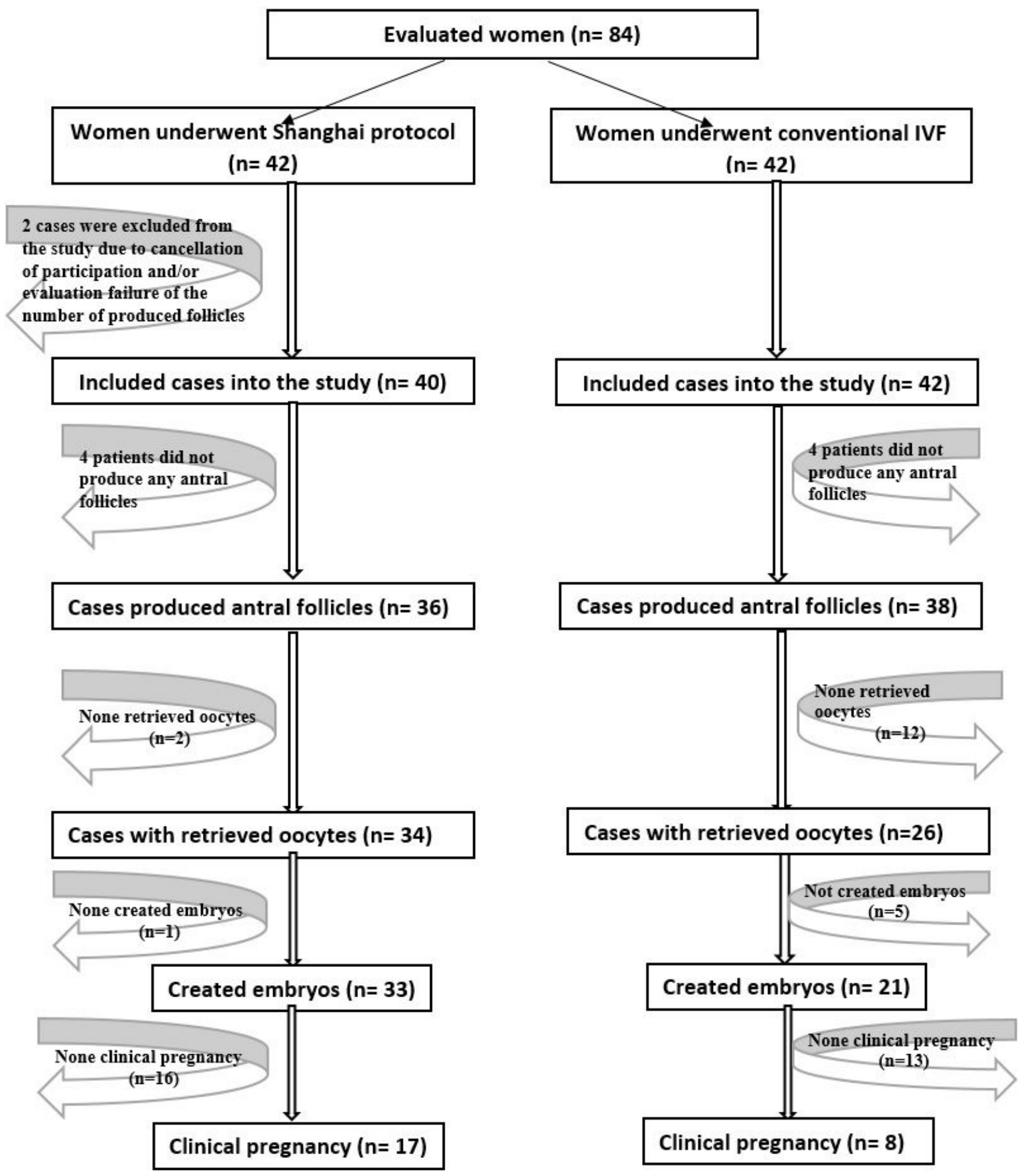

Figure 2

Evaluation of the quality of recovered oocytes from 34 patients showed that most of the recovered oocytes $(60.82 \%)$ were in metaphase stage II and there was an average of $1.63860 .86 \mathrm{MIl}$ oocytes. 